\title{
An Investigation into Plantar Pressure Measurement Protocols for Footwear
}

Research.

Authors: J.M.A. Melvin, S. Preece, C.J. Nester, D. Howard

Declaration regarding funding and role of RB:

The work in this paper was supported in part by funding by Scholl Footcare Brand (Reckitt Benkiser).

The company supported PhD studentship of J Melvin investigating the relationship between foot pressure and footwear designs. The work in this paper was carried out to support that work. No financial benefit was received by the authors for this work. Company representatives played no part in the work in this paper.

Corresponding author:

\section{Professor Chris Nester}

School of Health Sciences

PO 32 Brian Blatchford Building,

University of Salford,

Salford

M6 6PU

England.

t: +44 (0) 1612952275

c.j.nester@salford.ac.uk 


\section{Abstract}

Many researchers investigate how footwear design affects plantar pressure (PP) and ask participants to walk in unfamiliar footwear as part of their studies. However, there are no clear guidelines for the required period of time or number of steps a healthy participant requires to acclimatise to unfamiliar footwear. Nor are there clear guidelines for how many steps should be collected to produce data that is representative of gait in each particular shoe being tested. There were therefore two aims to this study: (1) to investigate the number of steps required to produce an average step that is representative of normal gait; (2) to investigate the number of steps required for a participant to acclimatise to a range of footwear types. PP data was collected in 20 healthy participants whilst they walked for $400 \mathrm{~m}$ in a range of footwear. The results showed that the number of steps required for both acclimatisation and to ensure data quality are dependent on shoe type and the foot region being investigated. It is recommended that 30 steps from one foot are collected during data collection and an acclimatisation period of at least 166 steps is given for each shoe condition. The former recommendation is not met by most studies in the literature.

Key words: Pedar; Plantar Pressure; Acclimatisation; Representative step 


\section{Introduction}

The magnitude and distribution of pressure on the plantar surface of the foot has been associated with foot pain [1, 2], comfort [1] and devastating clinical problems such as foot ulcers [3]. Footwear research has therefore sought to investigate specific footwear designs that might reduce plantar pressures (PP) [4-6]. A wide range of footwear styles have been investigated, including running shoes, high heeled shoes, boots, rollover footwear and instability footwear [4, 5, 7-11].

Many aspects of gait (PP [4], lower limb kinematics [12], kinetics [13] and muscle activity [14]) are sensitive to footwear design choices. Therefore, footwear can be said to represent a potential perturbation in the mechanical conditions under which gait is performed. Like any perturbation in the conditions of walking, some period of acclimatisation to the footwear is likely necessary and this is widely acknowledged by researchers in their protocols. Footwear that is similar to that worn by a participant on a daily basis might represent a small or indeed no perturbation at all, and require little acclimatisation. Investigation of some footwear styles however (e.g. stiletto, rollover footwear) might require more acclimatisation if participants are unfamiliar with their use.

Protocols used in prior research to acclimatise to footwear vary considerably and often only scant details are offered. Some studies require the participant to familiarise themselves with the study protocol rather than the footwear being tested[1, 15-17] and others had participants acclimatise to footwear walking on a treadmill but collected data during over ground walking [18]. Other investigators allow a period of time between conditions but do not explain the purpose of this period or what each participant did [19]. There are studies which allow the participant to acclimatise to each footwear condition $[20,21]$ and 5 minutes is the most common period of time provided. The variation in methodologies highlights the lack of understanding of the acclimatisation effect. Furthermore, no previous studies have reported quantitative criteria to define when a participant has acclimatised to a specific shoe design. 
A further issue regarding PP protocol design is how many steps are required to produce a valid representation of gait. Since no two steps during gait are the same [22] numerous steps must be measured and thereafter averaged to represent the individual participant and experimental condition. One study has suggested 200 steps are required when walking on a treadmill [23], although this was based on ground reaction rather than PP data. In PP studies it is typical to use fewer than 200 steps $[17,19,21,24]$.

The only comparable study based on PP data focussed on people with diabetes wearing rocker profile footwear [25]. Based on data from 20 steps, just 12 were required for valid data. However, people with diabetes exhibit different gait patterns and rocker footwear has features that are distinct from many footwear styles (very stiff sole, distinct angular sole profile). Thus, the generalizability of these data is low.

The two aims to this study are:

1) To identify the minimum number of steps required to produce an average which accurately represents normal gait.

2) To identify the minimum number of steps required for participants to acclimatise to a range of footwear types.

Our purpose in meeting these aims was to offer recommendations for future studies concerned with footwear effects on PP. 


\section{Method}

\section{Participants}

Twenty young adults (10 female, mean (SD) age $=28(7.1)$ years, height $=1.692 \mathrm{~m}(0.074)$; mass $=$ $69.9 \mathrm{~kg}(14.9))$ were each tested in one session. All were pain, injury and pathology free. The study was approved by the University ethics committee and all participants provided informed consent.

\section{Footwear}

Five footwear designs for females and 3 for males were selected to provide a range of perturbations and therefore possible acclimatisation periods and number of steps for valid data. For men: a Duna (Italy) diabetic rocker shoe with a $15^{\circ}$ rocker angle, a $55 \%$ apex position and a $80^{\circ}$ apex angle as defined by Chapman et al 2013[4]; Kalenji Ekiden 50 running shoe, and ; the Scholl Starlit instability shoe. In addition to the shoes worn by the males the females also wore: a stiff upper high heeled shoe (Scholl Flon, 55mm heel and bovine leather upper); a soft upper high heeled shoe (Scholl Flon, $55 \mathrm{~mm}$ heel and bovine suede upper) (Figure 1). 

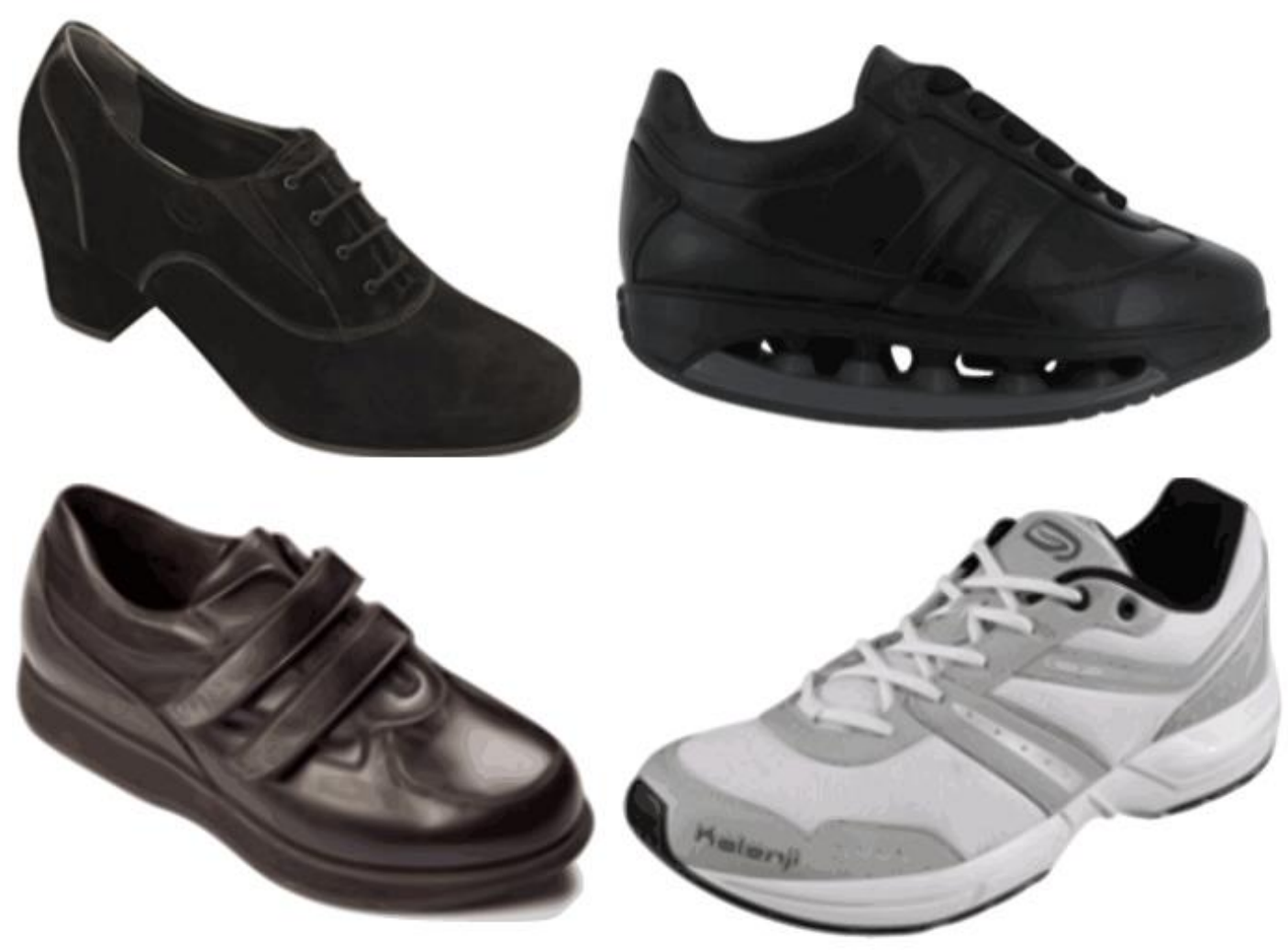

Figure 1: The shoes (Top left: Scholl Flon, Top right: Scholl Starlit, Bottom left: Duna Diabetic Rocker, Bottom right: Kalenji Ekiden 50)

\section{Protocol}

To familiarise themselves with the testing procedure participants walked in their own shoes for a period of five minutes up and down a $27 \mathrm{~m}$ walkway. We requested that the participant attend wearing a stable sports shoe and most wore running shoes whilst the remainder wore converse style shoes. The walkway comprised of a hard flat laminated surface and included a $20 \mathrm{~m}$ central area and a $3.5 \mathrm{~m}$ acceleration/ deceleration zone at each end. Participants were required to walk at a speed of $1.2 \mathrm{~m} . \mathrm{s}^{-1} \pm 5 \%$, which was measured using optical timing gates (Brower Timing Systems, Draper, Utah, USA). If we had allowed the participants to pick their own speed they may have chosen a speed that was too fast to comfortably walk in all shoe types. Since we could not let participants wear the test shoes before data collection, as this would have affected our acclimatisation results, we had to choose a speed we were confident all participants could walk at in all shoes. The high heeled shoes 
were thought most likely to affect walking speed and past studies had shown that participants are able to walk in excess of 1.2 meters per second in high heels [26]. During the familiarisation period, verbal feedback was provided to the participant until they could consistently walk at the correct speed. The testing was conducted in a laboratory setting and therefore it was not possible to collect $400 \mathrm{~m}$ of continuous walking. However, this study was designed to inform future protocols which are typically performed on a walkway of a similar length.

The Pedar (Novel) system was used to collect PP, however, this device can be affected by the heat and moisture in shoes [27]. Therefore, during the protocol and walking speed familiarisation period the Pedar insoles were placed inside the participant's own shoes to enable measurement sensors to acclimatise. After familiarisation the test shoes were tested in a random order (generated with a Matlab programme). When changing footwear the participant remained seated throughout and tied their laces and data collection started whilst participants were still seated. This ensured that data from every step taken in each shoe was collected and that there was no chance of missing the acclimatisation period if it was very short. Once stood, the participant walked the length of the collection area 20 times, thus completing $400 \mathrm{~m}$ of straight line walking for each shoe. Pilot tests indicated that this distance ensured that participants took more than the 200 steps recommended in past research [23]. Following data collection, the steps collected from the acceleration/ deceleration zones were removed from the dataset.

\section{Data analysis}

The data recorded by Pedar was divided into 6 areas as described by Cavanagh 1994 [28]: heel, midfoot, metatarsal phalangeal joint one (MTP1), metatarsal phalangeal joints 2-4 (MT24), Metatarsal phalangeal joint 5 (MT5), and hallux. It has been reported that peak, mean and impulse PP parameters are strongly correlated and need not be reported as independent parameters [24] 
[29]. As such, we chose to use the most commonly reported of all PP parameters (i.e. peak PP) to address our research questions.

In order to address our two research objectives, it was necessary to make a number of assumptions. The first was that a maximum of 200 steps would always be sufficient for a participant to acclimatise to an unfamiliar shoe condition. This figure was recommended in past research[23] and was well in excess of the vast majority of protocols used in the literature. The second assumption was that, following acclimatisation, no more than 60 steps would be required to calculate an average representative step. This value is ten more than previous research has indicated is necessary [23] and far more than most PP studies. Pilot testing showed that $400 \mathrm{~m}$ of walking was sufficient for participant to take at least 260 steps, 200 to ensure acclimatisation and a further 60 from which an average representative step could be calculated.

The first stage of the analysis was to identify the minimum number of steps which must be averaged to accurately represent normal gait following acclimatisation to footwear. This was achieved by comparing the peak pressure, averaged across the final 60 steps $\left(P_{\text {target }}\right)$ with the peak pressure averaged across a window spanning a smaller number of steps $\left(P_{\text {win }}\right)$. The width of $P_{\text {win }}$ was gradually increased until the absolute difference between $P_{\text {target }}$ and $P_{\text {win }}$ was within a tolerance of $2.5 \%$. This tolerance then had to be met for five consecutive steps (to ensure stability) before the size of the window was taken as the minimum number of steps required for a representation of normal gait. The final value reported was the first of the five consecutive steps to meet the criteria. This idea is illustrated in Figure 2 which shows the target pressure $P_{\text {target }}$ and two example windows of different widths ( $A$ and $B$ ). 


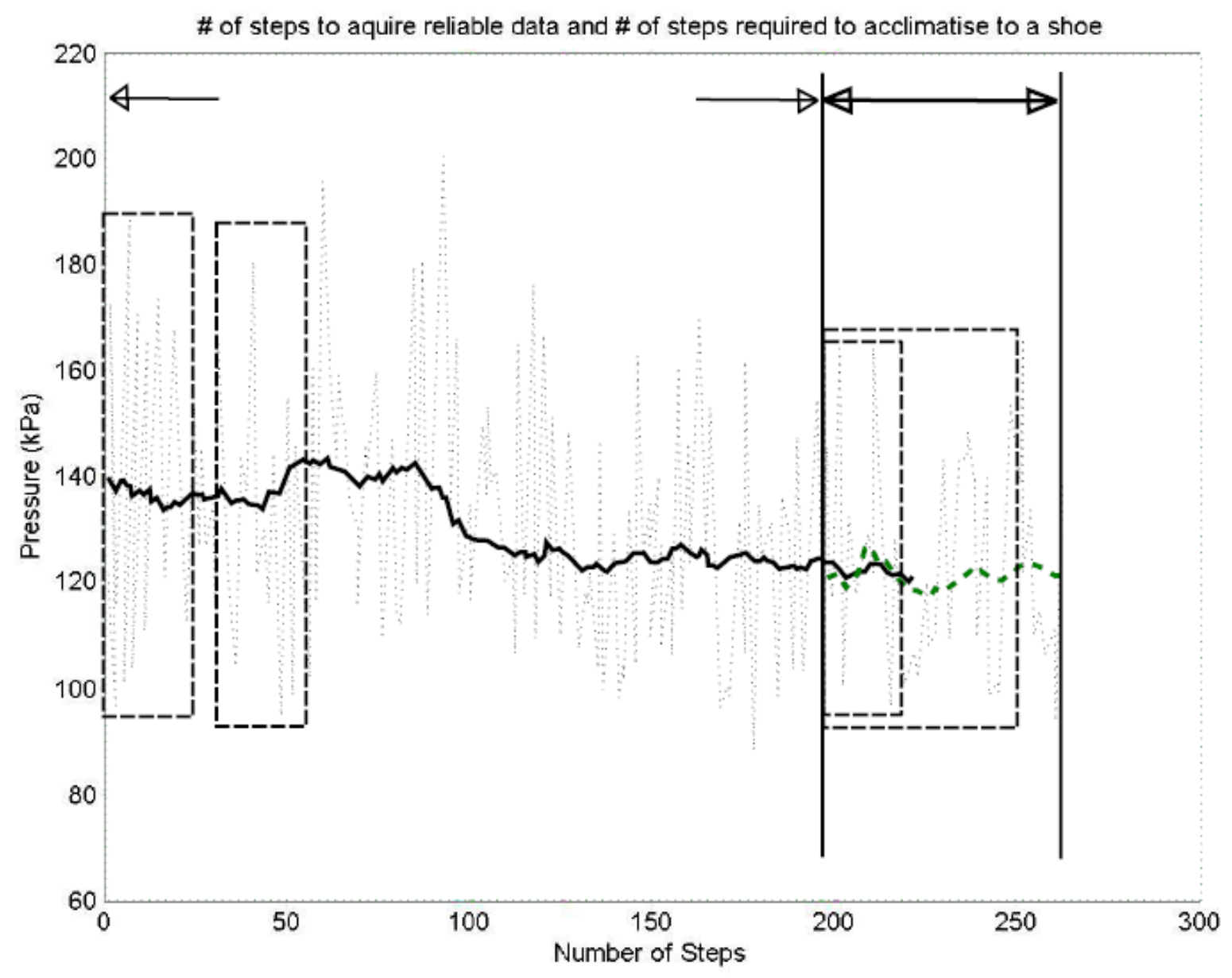

Figure 2: A depiction of the algorithms. Raw data (finely dashed line), last 60 steps cumulatively averaged used to produce $P_{\text {target }}$ (thick dash), Box $A$ shows the window used to calculate the cumulative mean $\left(P_{\text {win }}\right)$, Box B shows the same but for the later in the process and therefore includes more steps. Box $C$ shows window used to calculate the mean over 30 steps and this produces the first value used to produce the mean peak pressure (tick black line). This window then moves along one step and another average is taken to produce the thick line's second value. Box D shows the same as C but finds the mean peak pressure for a later step.

The processed described above was repeated for each participant, each shoe and each anatomical foot region. A mean (SD) number of steps was then obtained for each region and shoe across all the participants and the final recommendation calculated as the mean plus 2 SD across all participants. From these data a recommended minimum number of steps, or window size $\left(W_{\text {final }}\right)$, was then identified and this was used in the subsequent analysis (described below). 
The second stage of the analysis aimed to identify the number of steps required for participants to acclimatise to the different footwear types. This was achieved by gradually moving a window of width $\mathrm{W}_{\text {final }}$ in increments of 1 step along the peak pressure data (Figure 2). Note that Figure 2 shows two example windows at different positions ( $C$ and $D)$. For each window position, average peak pressure was calculated across the window and compared to the target pressure $\left(P_{\text {target }}\right)$ defined from the average of the final 60 steps (see above). Once the average peak pressure of the window was within $2.5 \%$ of $P_{\text {target }}$ it was assumed that the participant had fully acclimatised to the footwear. The number of steps at the start of the window was taken to be the end of the acclimatisation period. This process was repeated for each participant, each footwear condition and each region, each time using a window of width $\mathrm{W}_{\text {final }}$. A final recommendation for each footwear condition and region was then calculated as the mean plus 2SD across all participants.

Due to the known limitations of Pedar such as heat drift, resolution and hysteresis it was possible that the acclimatisation data may never be exactly equal to $P_{\text {target }}$. It was therefore deemed necessary to use a boundary within which an acceptable level of acclimatisation had been reached. To ensure a suitable boundary was chosen the above algorithms were run a number of times for a range of boundary sizes, and $2.5 \%$ was deemed the smallest acceptable. A comparison of the results when $2.5 \%$ and $5 \%$ boundaries were used can be seen in Figure 3. 


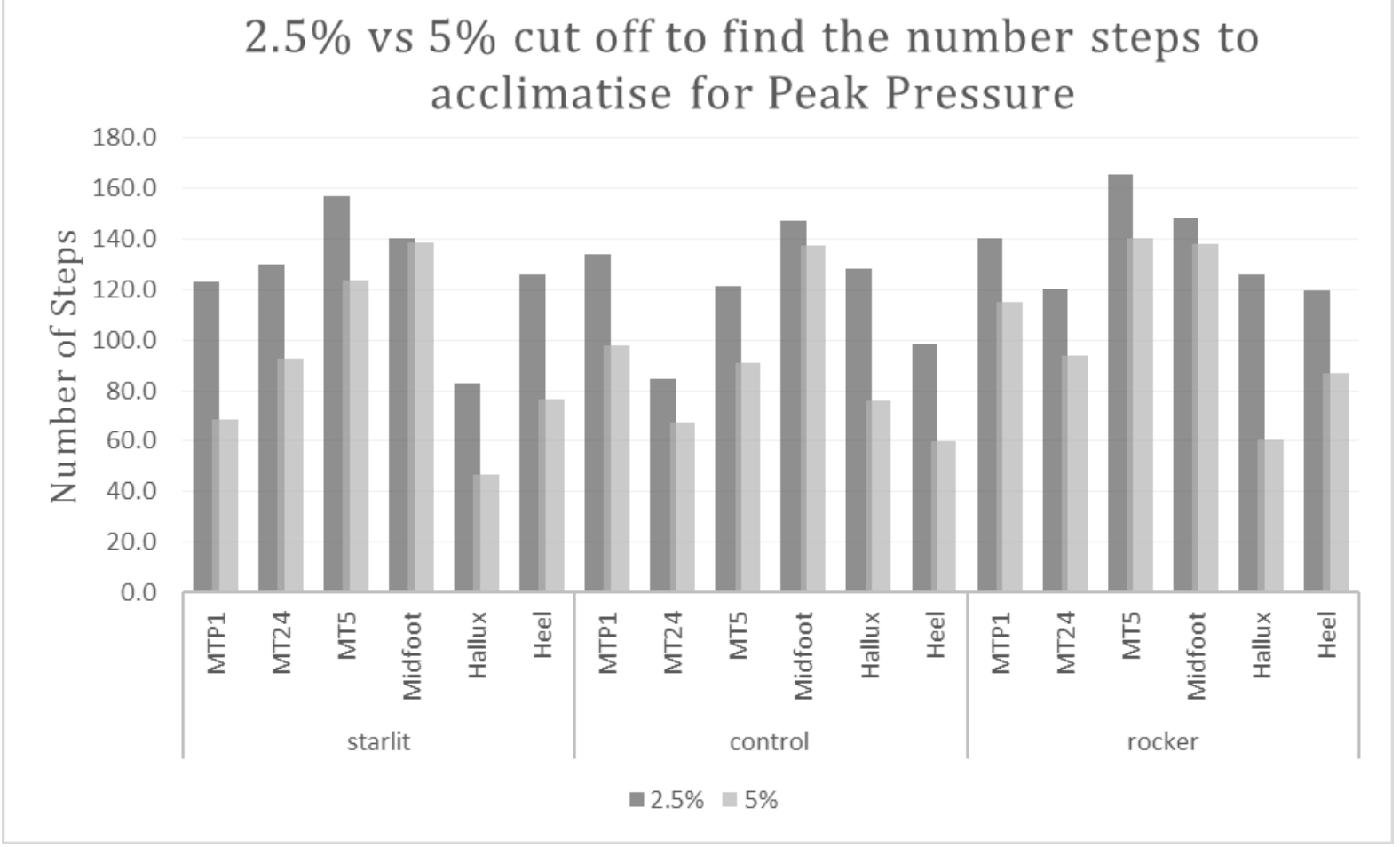

Figure 3: a graph showing the results for the number of steps required to acclimatise for 2.5 and $5 \%$ cut off from the average representative step established earlier, using peak pressure.

\section{$\underline{\text { Results }}$}

In females the highest minimum number of steps was 39 (MT5, soft upper heeled shoe) Table 1, lowest was 14 (hallux, Starlit and Rocker). In males, the highest minimum number of steps required to provide representative peak PP data was 34 (midfoot, Starlit shoe), the lowest was 2 (Hallux, Kalenji shoe). When considering both genders the foot area requiring the highest minimum number of steps was MT1 (27.9 steps, based both on shoes common to male and females) and the area requiring the least steps was MT24 (12.8 steps). The difference between the shoe requiring the highest and lowest minimum number of steps (averaged across all foot areas listed in table 1) was just 5 steps for the male shoes, and 13 steps for the female shoes. 


\section{Table 1}

Minimum number of steps required for reliable data, the value two standard deviations above the mean is presented

\begin{tabular}{llccccccc}
\hline Shoes & Gender & MTP1 & MT24 & MT5 & Midfoot & Hallux & Heel & Average \\
\hline starlit & both & 28.9 & 11.5 & 23.3 & 27.8 & 17.4 & 17.9 & 21.1 \\
Kalenji & both & 29.3 & 14.2 & 28.5 & 23.5 & 11.9 & 27.5 & 22.5 \\
rocker & both & 25.5 & 12.7 & 24.8 & 25.5 & 11.4 & 26.7 & 21.1 \\
Average & both & 27.9 & 12.8 & 25.5 & 25.6 & 13.6 & 24.0 & 21.6 \\
starlit & male & 32.2 & 10.3 & 23.7 & 33.9 & 20.4 & 14.8 & 22.6 \\
Kalenji & male & 26.9 & 12.7 & 33.3 & 15.6 & 1.3 & 32.3 & 20.4 \\
rocker & male & 21.2 & 11.0 & 22.4 & 25.0 & 8.2 & 28.1 & 19.3 \\
Stiff & female & 28.6 & 13.7 & 35.5 & 26.5 & 21.1 & 21.0 & 24.4 \\
Soft & female & 23.3 & 17.0 & 38.8 & 21.8 & 24.3 & 28.0 & 25.5 \\
Starlit & female & 25.2 & 13.2 & 24.2 & 21.8 & 13.4 & 20.0 & 19.6 \\
Kalenji & female & 33.1 & 16.0 & 22.4 & 28.6 & 15.3 & 22.6 & 23 \\
Rocker & female & 30.3 & 14.4 & 27.5 & 27.4 & 13.1 & 26.6 & 23.2 \\
\hline
\end{tabular}

\section{Acclimatisation period}

Based on the shoes common to both males and females, the longest acclimatisation period was 166 steps (MT5, Rocker shoe) and the shortest was 83 (Hallux, starlit). For the females, the longest acclimatisation period was 175 steps (midfoot, Kalenji shoe), shortest was 67 steps (Heel, Kalenji shoe). For the males, the longest acclimatisation period was 167 steps (midfoot, starlit shoe) and the shortest 56 steps (Heel, rocker shoe).

The foot area with the longest acclimatisation period was the MT5 (166 steps, based on male and female combined data), and the hallux had the shortest period ( 83 steps). The shoe with the longest acclimatisation period was the rocker shoe for females (144.2 steps averaged across all foot areas) and Starlit shoe for males (128.1 steps). The shoe with the shortest acclimatisation period was the soft upper heeled shoe for females (113.6 steps) and Kalenji shoe (111.2 steps) for the males. 
Table 2

The number of steps required to acclimatise to footwear using a 30-step window for comparison, the value two standard deviations above the mean is presented

\begin{tabular}{llccccccc}
\hline Shoes & Gender & MTP1 & MT24 & MT5 & Midfoot & Hallux & Heel & Average \\
\hline Starlit & Both & 123.3 & 129.8 & 157.1 & 140.3 & 82.8 & 126.1 & 126.6 \\
Kalenji & Both & 133.7 & 84.8 & 121.3 & 146.9 & 128.1 & 98.5 & 118.9 \\
Rocker & Both & 140.5 & 120.3 & 165.3 & 148.2 & 126.0 & 119.4 & 136.6 \\
Average & Both & 132.5 & 111.6 & 147.9 & 145.2 & 112.3 & 114.7 & 127.4 \\
Starlit & male & 113.7 & 120.5 & 151.0 & 166.3 & 73.1 & 144.1 & 128.1 \\
Kalenji & male & 113.6 & 87.4 & 109.6 & 120.5 & 113.4 & 122.7 & 111.2 \\
Rocker & male & 127.2 & 86.4 & 147.1 & 138.2 & 139.3 & 55.5 & 115.6 \\
Stiff & female & 116.5 & 131.8 & 142.3 & 140.9 & 104.5 & 113.2 & 124.9 \\
Soft & female & 103.5 & 78.9 & 134.7 & 123.0 & 148.8 & 92.9 & 113.6 \\
Starlit & female & 135.3 & 145.0 & 167.0 & 114.9 & 94.2 & 112.8 & 128.2 \\
Kalenji & female & 155.2 & 86.8 & 132.7 & 175.0 & 141.1 & 66.3 & 126.2 \\
Rocker & female & 137.4 & 151.7 & 152.1 & 153.3 & 115.3 & 155.3 & 144.2 \\
\hline
\end{tabular}

\section{Discussion}

We implemented a new approach to determine the minimum number of steps over which PP data should be recorded in order to provide valid peak PP data. Furthermore, we implemented a novel approach to establish the number of steps required to acclimatise to unfamiliar footwear. The values recommended for future research studies (30 steps for valid data, and 166 steps to acclimatise to footwear) are the upper boundaries of what is required. This is because we used the mean value +2 standard deviations to derive our recommendation which provide recommendations that include $95 \%$ of participants. In addition, these values represent worst case estimates because they are based on the highest values across all 6 foot regions and a varied range of shoes.

According to our data and the assumptions we made, the minimum number of steps required to produce an average step was 30 . This is far greater than the 12 steps suggested previously however this suggestion was based on study of the foot affected by diabetes and neuropathy [25]. As previously explained this may be because gait of those with diabetes is often slower and less variable 
than healthy individuals. Added to this, the prior study only used a 20 step protocol and thus could only have found that less than 20 steps are required due to the convergence of their data. The regions with the least variability and thus required the least steps to produce an average step, for both genders, was the MT2-4 followed by the hallux. These two regions represent those where high pressures are often reported $[18,19]$, and these high loads will make the small changes in loading less significant. Subsequently, the need for areas in the forefoot to adjust their loading may be reduced relative to that of the rear foot. This would lead to greater consistency in the pressure values and therefore fewer footsteps would need to be measured.

There was little effect due to the footwear design and thus we conclude that the number of steps required to produce valid PP data is not strongly dependent on the footwear. This might be surprising given the varied designs we chose, but it perhaps reflects the ability of the foot to quickly accommodate to new positions and external constraints.

According to our data and the assumptions we made, to acclimatise to an unfamiliar pair of shoes 166 steps were required. Males required fewer steps than females. For both genders it is the MTP5 and midfoot that required the most steps to acclimatise. Males acclimatised quickest in the Kalenji shoe, most likely because shoes of similar design are regularly worn by participants. The Starlit required the most steps in males, unsurprising given that this shoe was designed to create a significantly different and so called "unstable" interface [30] that none of the participants had prior experience of. By contrast, in females the Kalenji shoe required the most steps to acclimatise whilst the stiff high heel required the least. Further investigations are required to better understand why this is the case.

An important issue is whether prior research meets the recommendations we have proposed. The few papers that have reported an acclimatisation period for each footwear condition all provided 
sufficient opportunity for acclimatisation according to our results [17, 21]. However, our recommendation for 30 steps is greater than in many studies $[17,19,21]$. Indeed, only Wang and li 2005 recorded a sufficient number of steps (30 steps collected with a ten minute break between conditions) [19]. However, we would also concede that in some studies it may not be realistic to ask participants to perform 30 steps. For example, in cases of sensory neuropathy and diabetes and when investigating gait in older people, it might expose participants to risk if they are asked to walk too many steps in too many shoe conditions. However, arguably, the solution is to reduce the number of shoes in order to have better quality data.

This study has several limitations.

1. The assumption that 200 steps was more than necessary to acclimatise and that 60 steps were sufficient to acquire valid data are limitations. However, they are based on the findings of past research and represent assumptions that are commonly made in footwear and gait investigations. Whilst it could be argued that much longer periods are required to distinguish a macro gait cyclic pattern, doing this would not represent a useful and viable method of collecting data in future studies.

2. We investigated healthy participants rather than people affected by disease, which might alter step to step variability and increase the recommendations. However, the purpose of this study was to provide a base line for future investigations and therefore we adopted the upper second SD to widen the population covered by the recommendations.

3. The results are based on anatomical masks which can lead to a loss of data. To avoid this statistical mapping could be used [31], however, this is still not widely used in research investigations. 
In summary, it is recommended that the period required to acclimatise to unfamiliar footwear is 166 steps per foot and that no fewer than 30 steps of PP data should be recorded. 
[1] Jordan C, Bartlett R. Pressure distribution and perceived comfort in casual footwear. Gait \&amp; Posture. 1995;3:215-20.

[2] SILVINO N, EVANSKI PM, WAUGH TR. The Harris and Beath footprinting mat: diagnostic validity and clinical use. Clin Orthop Relat R. 1980;151:265-9.

[3] Frykberg RG, Harvey C, Lavery LA, Harkless L, Pham H, Veves A. Role of neuropathy and high foot pressures in diabetic foot ulceration. Diabetes Care. 1998;21:1714-9.

[4] Chapman JD, Preece S, Braunstein B, Hohne A, Nester CJ, Brueggemann P, et al. Effect of rocker shoe design features on forefoot plantar pressures in people with and without diabetes. Clin Biomech (Bristol, Avon). 2013;28:679-85.

[5] Lee YH, Hong WH. Effects of shoe inserts and heel height on foot pressure, impact force, and perceived comfort during walking. Applied Ergonomics. 2005;36:355-62.

[6] van Schie C, Ulbrecht JS, Becker MB, Cavanagh PR. Design criteria for rigid rocker shoes. Foot Ankle Int. 2000;21:833-44.

[7] Cheng YL, Hong Y. Using size and pressure measurement to quantify fit of running shoes.

Footwear Science. 2010;2:149-58.

[8] Cowley EE, Chevalier TL, Chockalingam N. The Effect of Heel Height on Gait and Posture A Review of the Literature. J Am Podiat Med Assn. 2009;99:512-8.

[9] Mandato MG, Nester E. The effects of increasing heel height on forefoot peak pressure. J Am Podiat Med Assn. 1999;89:75-80.

[10] Crenshaw SJ, Pollo FE, Brodsky JW. The effect of ankle position on plantar pressure in a short leg walking boot. Foot Ankle Int. 2004;25:69-72.

[11] Stewart L, Gibson J, Thomson CE. In-shoe pressure distribution in "unstable"(MBT) shoes and flat-bottomed training shoes: a comparative study. Gait \& Posture. 2007;25:648-51.

[12] Opila-Correia KA. Kinematics of high-heeled gait with consideration for age and experience of wearers. Archives of physical medicine and rehabilitation. 1990;71:905-9.

[13] Esenyel M, Walsh K, Walden JG, Gitter A. Kinetics of high-heeled gait. J Am Podiatr Med Assoc. 2003;93:27-32.

[14] Kerrigan DC, Todd MK, Riley PO. Knee osteoarthritis and high-heeled shoes. Lancet. 1998;351:1399-401.

[15] Snow RE, Williams KR, Holmes GB, Jr. The effects of wearing high heeled shoes on pedal pressure in women. Foot \& Ankle. 1992;13:85-92.

[16] Queen RM, Abbey AN, Wiegerinck JI, Yoder JC, Nunley JA. Effect of shoe type on plantar pressure: A gender comparison. Gait \& Posture. 2010;31:18-22.

[17] Stöggl T, Haudum A, Birklbauer J, Murrer M, Müller E. Short and long term adaptation of variability during walking using unstable (Mbt) shoes. Clinical Biomechanics. 2010;25:816-22.

[18] Yung-Hui L, Wei-Hsien H. Effects of shoe inserts and heel height on foot pressure, impact force, and perceived comfort during walking. Applied Ergonomics. 2005;36:355-62.

[19] Wang L, Li J. Gait Characteristics and Pressure Distribution For Barefoot and Various Heel Height Shoes During Walking. ISBS-Conference Proceedings Archive2008.

[20] Wegener C, Burns J, Penkala S. Effect of Neutral-Cushioned Running Shoes on Plantar Pressure Loading and Comfort in Athletes With Cavus Feet A Crossover Randomized Controlled Trial. The American journal of sports medicine. 2008;36:2139-46.

[21] Hong WH, Lee YH, Chen HC, Pei YC, Wu CY. Influence of heel height and shoe insert on comfort perception and biomechanical performance of young female adults during walking. Foot Ankle Int. 2005;26:1042-8.

[22] Putti AB, Arnold GP, Cochrane L, Abboud RJ. The Pedar (R) in-shoe system: Repeatability and normal pressure values. Gait \& Posture. 2007;25:401-5.

[23] Owings TM, Grabiner MD. Measuring step kinematic variability on an instrumented treadmill: how many steps are enough? Journal of Biomechanics. 2003;36:1215-8.

[24] Chen H, Nigg BM, de Koning J. Relationship between plantar pressure distribution under the foot and insole comfort. Clin Biomech. 1994;9:335-41. 
[25] Arts ML, Bus SA. Twelve steps per foot are recommended for valid and reliable in-shoe plantar pressure data in neuropathic diabetic patients wearing custom made footwear. Clin Biomech (Bristol, Avon). 2011;26:880-4.

[26] Lee $\mathrm{YH}$, Hong WH. Effects of shoe inserts and heel height on foot pressure, impact force, and perceived comfort during walking. Applied ergonomics. 2005;36:355-62.

[27] Barnett S, Cunningham JL, West S. A Comparison of vertical force and temporal parameters produced by an in-shoe pressure measuring system and a force platform. Clin Biomech.

2001;16:353-7.

[28] Cavanagh P, Ulbrecht J. Clinical plantar pressure measurement in diabetes: rationale and methodology. The foot. 1994;4:123-35.

[29] Keijsers NLW, Stolwijk NM, Pataky TC. Linear dependence of peak, mean, and pressure-time integral values in plantar pressure images. Gait \& Posture. 2010;31:140-2.

[30] Forghany S, Nester CJ, Richards B. The effect of rollover footwear on the rollover function of walking. J Foot Ankle Res. 2013;6:24.

[31] Pataky TC, Caravaggi P, Savage R, Crompton RH. Regional peak plantar pressures are highly sensitive to region boundary definitions. Journal of Biomechanics. 2008;41:2772-5. 